\title{
Erratum: Doublon lifetimes in dissipative environments [Phys. Rev. B 96, 045408 (2017)]
}

\author{
M. Bello $\odot$, G. Platero, and S. Kohler
}

(Received 8 October 2019; published 21 October 2019)

DOI: 10.1103/PhysRevB.100.169905

Figures 3-6 contain wrong axes, having factors of $\times 4$ or $\times 1 / 4$ missing, depending on the case. These errors do not affect the results and conclusions of this paper. Below, we specify the correction for each figure:

(i) In Fig. 3, the abscissa axis should be multiplied by a factor of $\times 4$. The correct figure is

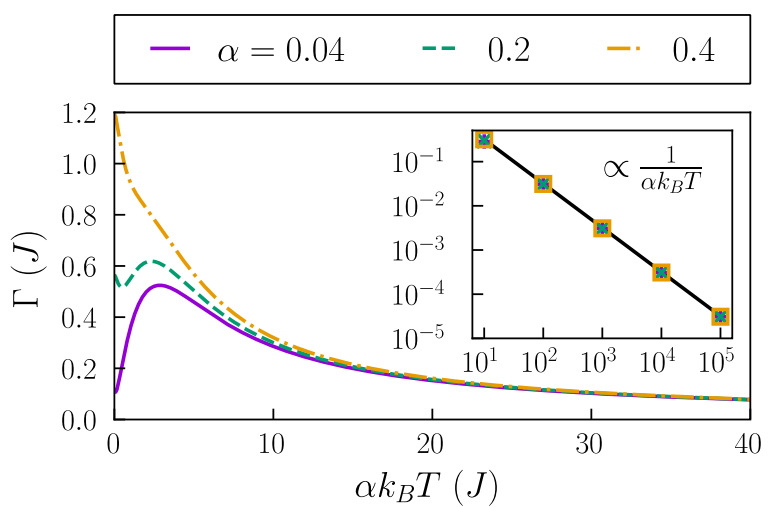

FIG. 3. Temperature dependence of the numerically obtained decay rate for a chain with $N=5$ and periodic boundary conditions in the presence of charge noise. The interaction energy is set to $U=10 \mathrm{~J}$. For values of the coupling strength $\alpha \lesssim 0.04$, we obtain approximately the same curve (continuous line). (The inset) Values for $\Gamma$ on a logarithmic scale demonstrating the proportionality $\propto 1 / \alpha T$.

(ii) In Figs. 4(a) and 4(b), the ordinate axis should be multiplied by a factor of $\times 1 / 4$. The correct figure is
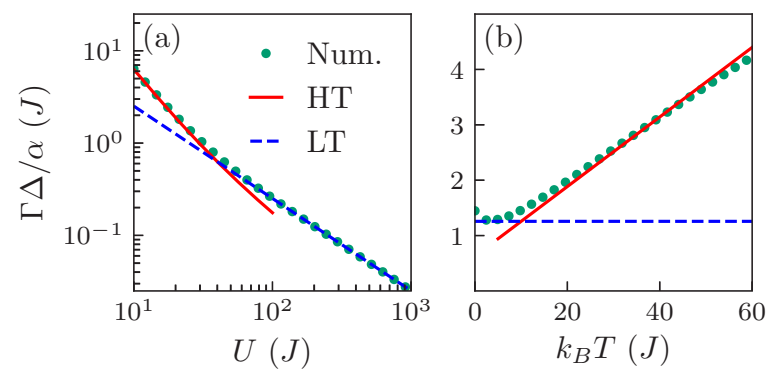

FIG. 4. Comparison between the numerically computed decay rate and the analytic formulas (17) and (18) for a chain with $N=5$ sites and periodic boundary conditions in the case of charge noise. The dissipation strength is $\alpha=0.02$. (a) Dependence on the interaction strength for a fixed temperature $k_{B} T=20 \mathrm{~J}$. (b) Dependence on the temperature for a fixed interaction strength $U=20 \mathrm{~J}$. 
(iii) In Fig. 5, the abscissa axis should be multiplied by a factor of $\times 4$. The correct figure is

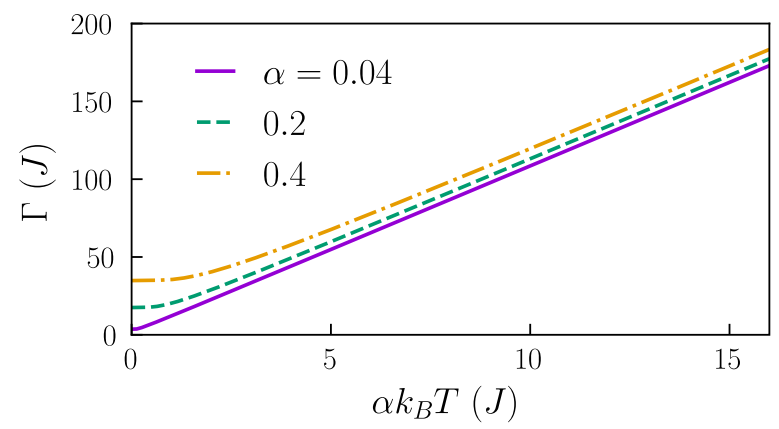

FIG. 5. Average decay rate of the doublon states under the influence of current noise for various dissipation strengths as a function of the temperature. The chain consists of $N=5$ sites with periodic boundary conditions, whereas the interaction is $U=10 \mathrm{~J}$.

(iv) In Figs. 6(a) and 6(b), the ordinate axis should be multiplied by a factor of $\times 1 / 4$. The correct figure is

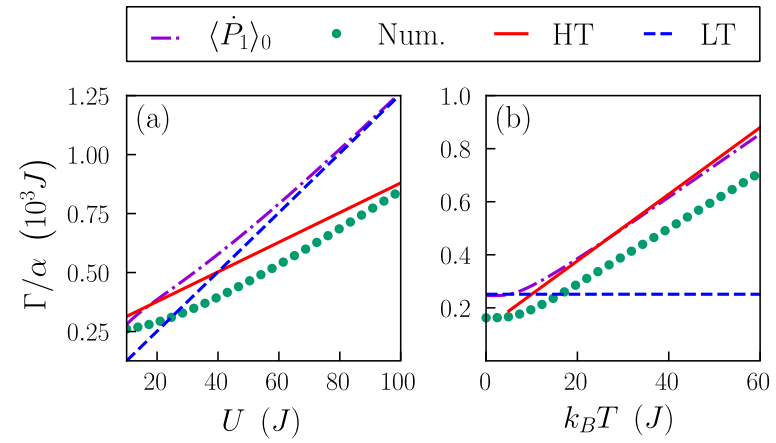

FIG. 6. Numerically obtained decay rate in comparison with the approximations (20)-(22) for a chain with $N=5$ sites and periodic boundary conditions in the case of current noise with strength $\alpha=0.02$. The results are plotted as a function of (a) the interaction and the temperature $k_{B} T=20 \mathrm{~J}$ and (b) for a fixed interaction $U=20 \mathrm{~J}$ as a function of the temperature.

The origin of these mistakes can be traced back to the first version of the paper. We initially used $\mathcal{J}(\omega)=2 \pi \alpha \omega$ for the spectral density, which is a customary expression used in several important references [1]. However, in the last version of the paper, we changed it to $\mathcal{J}(\omega)=\pi \alpha^{\prime} \omega / 2$, in accordance with other references [2]. Both lead to the same dynamics as long as $\alpha^{\prime}=4 \alpha$, so we kept using the same data changing the value of the dissipation strength accordingly in the captions of the figures. However, we missed the fact that some axes correspond to variables multiplied or divided by $\alpha$.

[1] H. Breuer and F. Petruccione, The Theory of Open Quantum Systems (Oxford University Press, Oxford, 2007).
[2] F. Forster, G. Petersen, S. Manus, P. Hänggi, D. Schuh, W. Wegscheider, S. Kohler, and S. Ludwig, Phys. Rev. Lett. 112, 116803 (2014). 